\title{
Performance Evaluation of Power Aware VM Consolidation using Live Migration
}

\author{
Gaganpreet Kaur Sehdev \\ Guru Nanak Dev University/CSE department, Amritsar, 143001, India \\ Email:gagansehdev204@gmail.com \\ Anil Kumar \\ Guru Nanak Dev University/CSE department, Amritsar, 143001, India \\ Email: anil.gndu@gmail.com
}

\begin{abstract}
Power Efficiency is the efficient use of power and is a crucial come forth in cloud computing environment. Green Computing is nothing but is a cloud computing with efficient use of power and green refers to make the environment friendly to the user by saving heat and power. Data centre power efficiency in cloud environment will be reduced when virtualization is used as contrary to physical resource deployment to book adequate to grant all application requests. Nevertheless, in any case of the resource provisioning approximation, occasion remains in the way in which they are made attainable and workload is scheduled. The objective of this research work is therefore to pack workload into servers, selected as a function of their cost to operate, to achieve (or as close to) the utmost endorsed employment in a cost-efficient manner, avoiding occurrences where devices are under-utilized and management cost is acquired inefficiently. This work has enhanced the existing work by introducing the dynamic wake up calls either to shut down the active servers or restart the passive server. The wakeup calls has been initiated dynamically. The overall objective is to decrease the response time of users which will be increased during wakeup time in existing research work.
\end{abstract}

Index Terms - Cloud Computing, Green Computing, Power Efficiency, Live Migration, Wake-up Calls, Swap in and swap out.

\section{INTRODUCTION}

Cloud is Common, Location-independent, Online Utility provisioned on-Demand. The term "cloud" is being created from the world of long distance communication when providers began using virtual private network (VPN) services for data communications. Amazon, Salesforce, Google, Microsoft and IBM are the major cloud computing service providers [2] [7]. Green IT is an upcoming technology for achieving efficient processing and utilization of computing infrastructure, and also it minimizes the energy consumption Figure1. The field of green computing is clarified as "the knowledge and practice of designing, manufacturing, using, and disposing of computers, servers, and associated subsystems-which include networking, storage devices and communications systems - efficiently and effectively with minimal or no impact on the environment [40]. In individual characteristics, Cloud resources need to be allocated not only to satisfy QoS requirements specified by users via Service Level Agreements (SLA), but also to decrease energy employment [16][23].

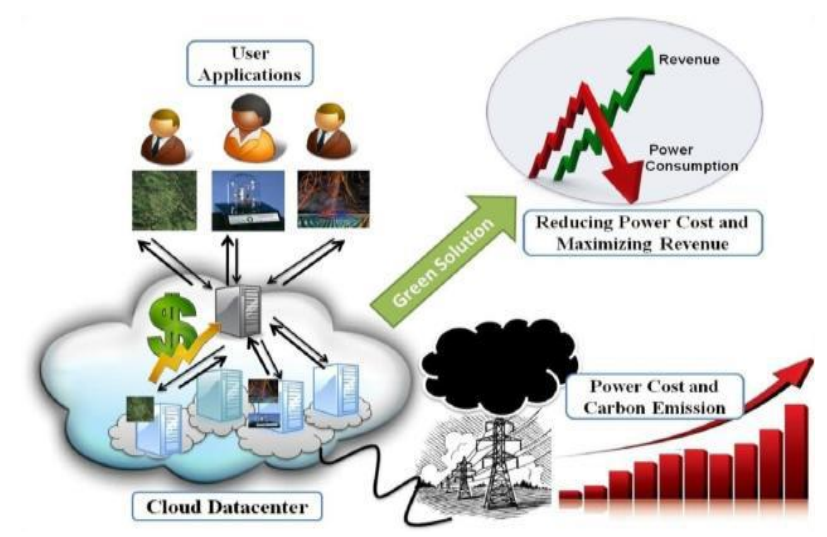

Fig. 1: Cloud and Environmental Sustainability [19]

\section{RELATED WORK}

With the entrance of internet in the 1990s to the present day facilities of ubiquitous computing, the internet has changed the computing world in a drastic way [2]. Cloud computing is a latest trend in information technology that moves computing and data away from desktop and portable PCs into large data centers.

With the deep knowledge of the concept of virtualization the concepts of ISA, API and ABI interfaces are discussed in detail [6]. ISA (Instruction Set Architecture) provides an interaction between application program and hardware. API (Application Programming Interface) provides interface between applications and ABI (Application Binary Interface) acts as boundary between software and hardware. It also discusses the compatibility issues of processors supporting ISA with some Operating systems. It provides with a clear definition about the guest, the Host and Virtual Machine Monitor [6]. It also discusses about the emulation of one 
instruction set with another, replicating VMs so that multiple OS instances can be run simultaneously and composing VM software to form a more complex flexible system.

Green technology is the environmentally sustainable technology and there are use of computers and related resources like - monitors, printer, storage devices, networking and communication systems - efficiently and effectively with minimal or no impact on the environment. Its goals are discussed in which there is to reduce the use of hazardous materials, maximize energy efficiency during the product's lifetime, and promote the recyclability or biodegradability of defunct products and factory waste.

Computers today not only used in offices but also at homes. As the number of computers is increasing day by day, so is the amount of electricity consumed by them which in turn is increasing the carbon content in atmosphere. This problem has been realized by people and measures are being taken which help in minimizing the power usage of computers [12]. Therefore, this can be called as Green Computing.

The pervasive use of cloud computing and the resulting rise in the number of datacenters and hosting centers have brought forth many concerns including the electrical energy cost, peak power dissipation, cooling, carbon emission, etc. [15].

The energy overhead of migration is much reduce in the scripts of employing the strategy of consolidation than the regular deployment without using consolidation and the results are based on the typical physical server, the power of which is linear model of CPU utilization percentage. The survey shows that management techniques alters to different types of servers and their associated workloads can provide substantial energy savings with little or no performance degradation [28].

At present environmental challenge is global warming. Various approaches to the green IT are virtualization, Power management, recycling and telecommunicating [29]. The result in this study should not have only a direct impact on the reduction of electricity bills of cloud infrastructure providers, but also imply possible savings in other operational cost of course the reduction in the carbon footprint of clouds is another important spinoff . VMM schedulers have relied on fairly sharing the processor resources among domains while leaving the scheduling of I/O resources as a secondary concern. This paper is the first to study the impact of the VMM scheduler on performance using multiple guest domains concurrently running different types of applications [30]. This cross product of scheduler configurations and application types offers insight into the key problems in VMM scheduling for I/O and motivates future innovation in this area.

Dynamic server consolidation through live migration is an efficient way towards energy conservation in Cloud data centers. The main objective is to keep the number of power-on systems as low as possible and thus reduce the excessive power used to run idle servers [35]. This technique of VM live migration is being used widely for various system-related issues like load balancing, online system maintenance, fault tolerance and resource distribution. Energy efficient VM migration becomes a main concern as the data centers are trying to reduce the power consumption.

Virtualization is applied across Information Technology (IT) infrastructure. VM migration improves the performance of the overall system by effectively balancing the load across servers. However, energy consumption is not considered in most of the migration algorithms [38]. The number of VM's and various other important factors are used in selecting VMs to migrate such that the energy consumption is minimized by switching off underutilized servers. This reduces the energy cost, including the cooling cost at datacenters. Cloud computing is a new technology which is proffering IT services based on pay-as-you-go model to consumers from everywhere in the world. The growing demand of Cloud infrastructure and modern computational requests like business, scientific and web applications result in large-scale data centers and lead to extra electrical energy consumption. High energy consumption causes high operational cost and also led to high carbon emission which is harmful for atmosphere [39]. Virtual machines (VMs) migration, dynamic consolidation in the virtualized data centers in cloud environments and switching idle physical machines off could yield reduce energy consumption; hence, one of the important issues for energy efficiency in virtualized cloud environments is how to place new VMs or selected VMs for migration across the hosts.

Cloud Computing is a utility computing and is concerned with IT services. It enables hosting of applications from consumers, scientific and business domains. However data centers hosting cloud computing applications consume huge amounts of energy, contributing to high operational costs and carbon footprints to the environment [41].

With energy shortages and global climate change leading our concerns these days, the power consumption of data centers has become a key issue. Therefore, there is a need of green cloud computing solutions that can not only save energy, but also reduce operational costs. Today many applications were moved to Cloud and the Cloud computing became more popular. For being able to satisfy all the client's resource demands, the Cloud service providers are continuously increasing their data centers. The recent studies has shown that a data center can be very costly due to the energy power consumption so the cloud service providers may have issues in cost recovering [42]. There are many resources that are not used at their maximum capacity or even worst, there are many resources that are not used at all.

\section{RESEARCH MOTIVATION}

The data centers consist of thousands of heterogeneous servers that consume lots of power and produce large amounts of heat [13]. Green scheduling systems for Data 
centers consists of: Power-aware and Thermal- aware Scheduling.

a. In thermal-aware scheduling, jobs are scheduled in a manner that minimizes the overall data center temperature.

b. In power-aware scheduling [33], jobs are scheduled to nodes in such a way to minimize the server's total power. The largest operating cost incurred in a Cloud data center is in operating the servers. So, concentrate is on power-aware scheduling in this research.

Virtualization is an advance way to abstract the hardware and system resources from an operating system. The aim of virtual computing environment is to improve resource employment by providing operating platform for users and applications based on mass of heterogeneous and autonomous resources. Cloud computing already leverages virtualization for load balancing via dynamic provisioning and migration of virtual machines (VM or guest in the following) among physical nodes. Virtual machines are allowed to share the resources of the host machine but still can provide separation between VMs and between the VMs and the host [5].

Nowadays with the arrival of processors having multiple cores like Intel Dual Core and Core 2 Duo, allows for a consolidation of resources within any data center. It is the Cloud provider's job to fully utilize this capability to its maximum potential while still maintaining a given QoS. "Fig. 2" and "Fig. 3" illustrate the motivation behind power-aware VM scheduling.

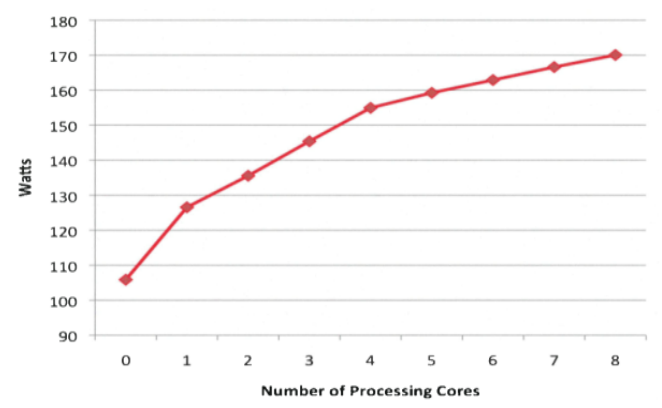

Fig. 2: Power consumption curve of an Intel Core i7 920 CPU [13]

The Intel Core i7 $920 \mathrm{CPU}$ has 8 cores. VM can be build upon every single core, so this means we can have 8 VMs running on every core of Intel Core i7 $920 \mathrm{CPU}$ [13].

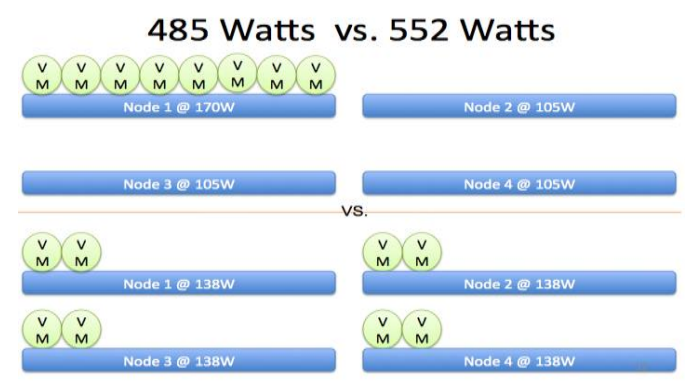

Fig. 3: Illustration of power savings [13]

The power consumption curve illustrates that as the number of processing cores increases, the amount of energy used does not increase proportionally. In fact the change in power consumption decreases. When only one processing core is used, the change in power consumption incurred by using another processing core is over 20 watts. The change from 7 processing cores to all 8 processing cores results in an increase of only 3.5 watts. The impact of this finding is substantial [13].

\section{A. VM migration}

VM live migration is that in which a VM is transferred from a physical server to another while continuously running, without any noticeable effects from the point of view of end users [21]. Multiple VMs can be dynamically started and stopped on a physical machine providing elasticity and flexibility. Also the VMs can migrate across physical machines so workloads can be consolidated and also unused resources can be turned off or switched to low-power [39][41].
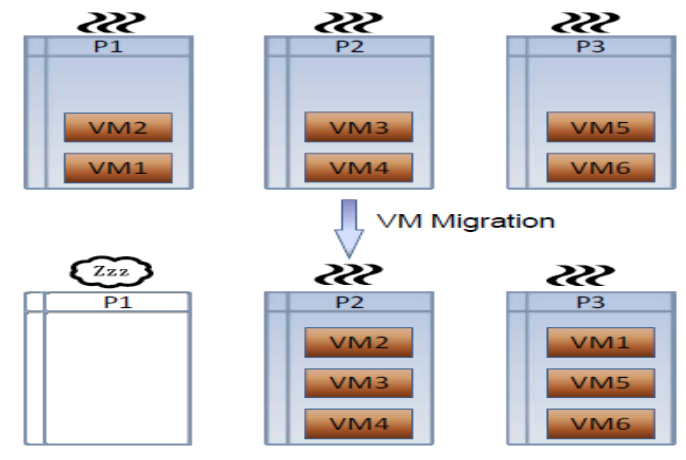

Fig. 4: Example of VM Migration [46]

It consists of $6 \mathrm{VMs}$ (VM1-VM6) running on three physical servers (P1-P3) with the VM migration technology. VM1 and VM2 consolidated on P2 and P3 respectively. And P1 is turned off, so the power consumption of the cluster is reduced [21].

\section{RESEARCH MODEL}

To attain the objective, step-by-step methodology is used in this research. This research work starts with the orientation in the area of cloud computing. 


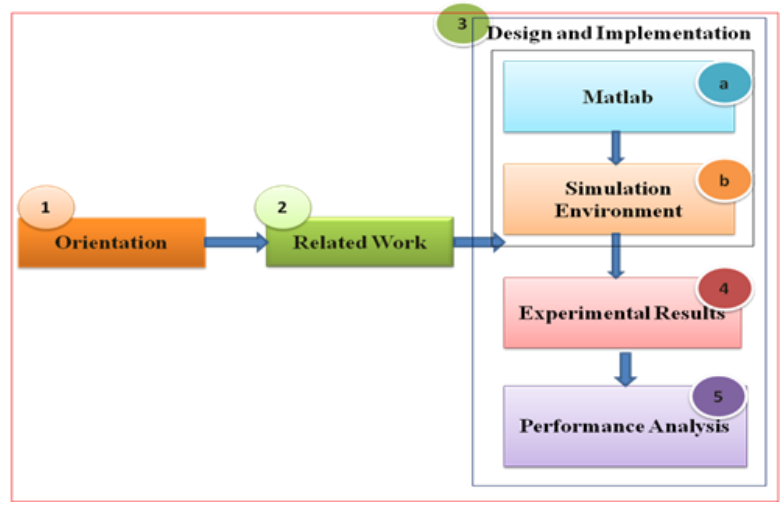

Fig. 5: Research model 
This research employs a structured method to obtain high quality information, called a related work.

\section{PROPOSED AlgorithM}

In the proposed algorithm as if active load and current load combinely is greater than the 80 percent then there is a generation of wake up call. Then find the best server out of all the three servers according to the priority assigned to the servers. After that if all the servers are free then the server with the lowest probability or load is used. The active load is incremented and at last as if active load and current load combinely is greater than the server's capacity then migration of the current load to available server has been taken place.

\section{Proposed Algorithm}

1. If (Active load + Current load) $>=80 \%$ then

\section{a) Generate Wakeup call}

2. Now, find the best server

3. If all servers are free then servers with lowest probability will be used

4. Active++

5. If (Active load+ Current load required) $>=$ Server capacity then

a) Migrate Current load to available server

\section{Flowchart of the proposed algorithm:}

In "Fig. 6" the flowchart of proposed algorithm is mentioned. There are certain steps to understand the proposed work in which response time and waiting time produces much better results as compare to earlier work. The wakeup call is initiated when the load is overloaded. Live migration concept is applied to migrate the virtual machine from one to other server.

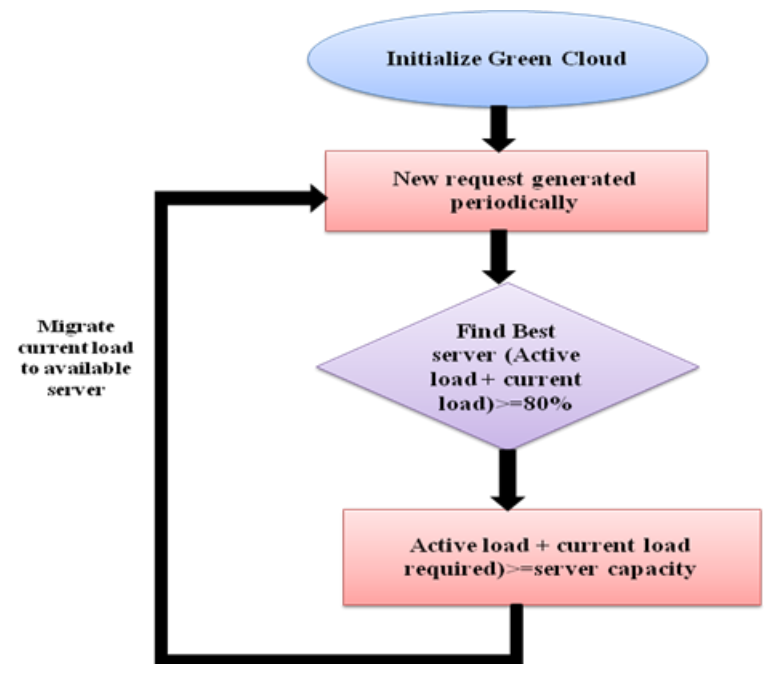

Fig. 6: Flowchart of Proposed Algorithm

Step 1. Firstly, initialize the green cloud.

Step 2. Then new request is generated periodically.
Step 3. Find best server as if active load and current load is greater than $80 \%$ then wakeup calls are generated.

Step 4. And if active load and current load is greater than equal to server capacity then migrate the current load to available load.

\section{SiMULATION ENVIRONMENT}

In order to implement the proposed fail-over strategy a suitable simulation environment has been made as demonstrated in "Fig. 7".

CSP is a cloud service provider that provides the interaction and services to the clouds says servers as by requested by the users.

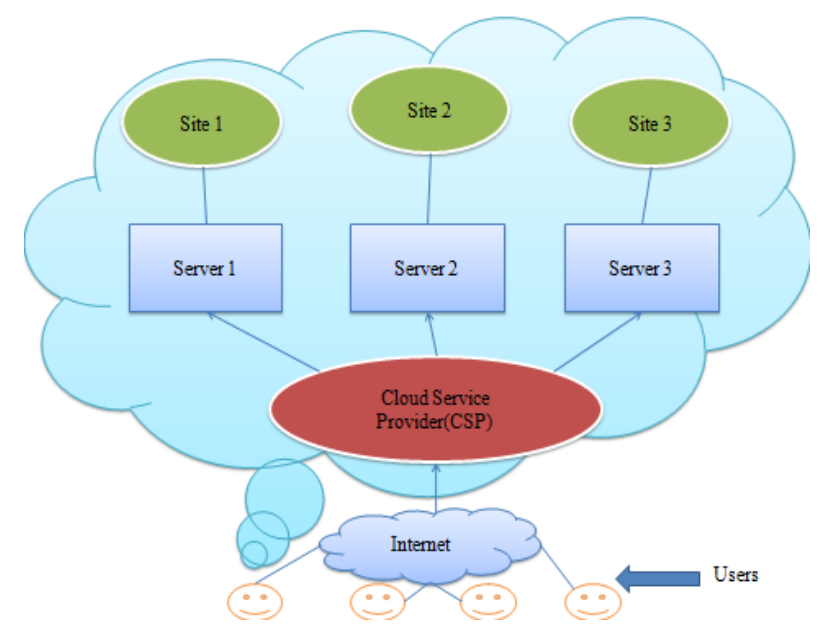

Fig. 7: Simulation Environment for proposed work

In simulation environment of proposed work there are three servers refers to $\mathrm{S} 1, \mathrm{~S} 2$ and $\mathrm{S} 3$. S1 refers to Server1 and $\mathrm{S} 2$ to server2 and $\mathrm{S} 3$ to server3. Also there exists a middleware which generally refers to CSP.

\section{PERformance ANALYSIS}

In this comparison table, failover is defined as if in any case if any cloud fails and the migration concept is not properly discussed and is overcome in proposed method. Live migration is the process of migrating one virtual machine to another and is present in existing as well as proposed work. Middleware is the cloud service provider and is like an intermediatory as actual work is done by server but it is defined by the CSP (Cloud Service Provider). Dynamic wakeup call concept is not mentioned in existing work but is overcome in proposed work. Response Time and waiting time both decreases in proposed work and the concept of swap in and swap out that is granularity is good in proposed work. The overall comparison shows clearly that the proposed work is much better than the existing work as it overcomes the limitations (gaps) of existing work. 
Table 1: Comparison Table of Existing work and Proposed work

\begin{tabular}{|c|c|c|}
\hline Parameters & Existing Work & Proposed Work \\
\hline Failover & No & Yes \\
\hline Live Migration & Yes & Yes \\
\hline Middleware & Yes & Yes \\
\hline $\begin{array}{c}\text { Dynamic Wakeup } \\
\text { call }\end{array}$ & No & Yes \\
\hline Response Time & Average & Good \\
\hline Waiting Time & Average & Good \\
\hline Granularity & Average & Good \\
\hline
\end{tabular}

1. Granularity is how much time system has taken for computational task and how much taken for extra computation like swap in and swap out etc.

2. Failover is defined a procedure by which a system automatically transfers control to a duplicate system when it detects a fault or failure.

3. Waiting Time is defined as amount of time a process has been waiting in the ready queue.

4. Response time is amount of time it takes from when a request was submitted until the first response is produced, not output (for time-sharing environment). It is the time between submission of requests and first response to the request.

5. Middleware (CSP) is a simple middleware definition: software that connects computers and devices to other applications. It is an abstraction layer that hides detail about hardware devices or other software from an application.

6. Live Migration refers to the process of moving a running virtual machine or application between different physical machines without disconnecting the client or application.

7. Dynamic Wakeup Call is used when load increases then we use dynamic wakeup call to start server back up.

\section{EXPERIMENTAL RESULTS}

In the proposed work the simulation time is to be given into the simulator and simulator responds according to that time. Here the figures shown below are the results for existing work and proposed work. Here the comparison is shown in behalf of response time, waiting time, capacity evaluation and load evaluation.

1. Case 1: Experimental Results for Existing work when simulation time is 55 :

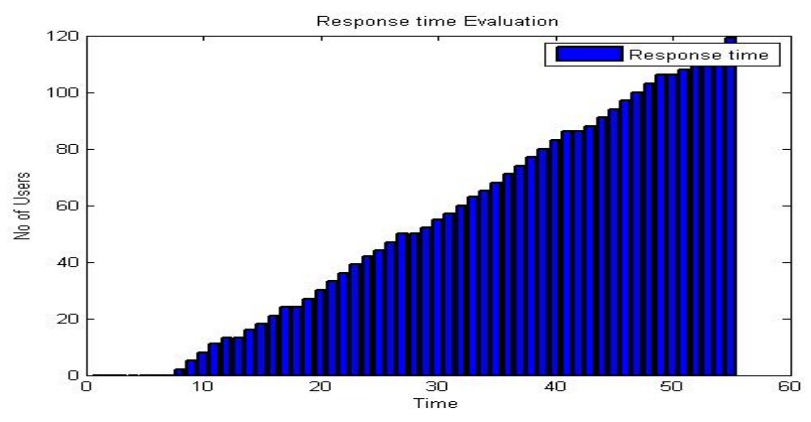

Fig. 8: Response Time Evaluation (Simulation time $=55$ )
The experimental results for existing work with simulation time 55 are shown below. "Fig. 8" shows the response time evaluation of the existing work where $y$ axis contains the number of users with respect to the time. It clearly shows that as the time increases, response time is also increases at a rapid rate due to the limited resources.

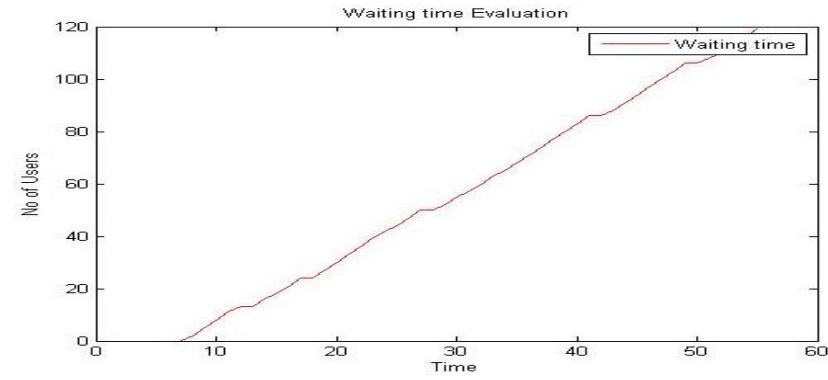

Fig. 9: Waiting Time Evaluation (Simulation time =55)

"Fig. 9" shows the waiting time evaluation of the existing work where y-axis contains the number of users with respect to the time. It shows that as the simulation time increases, waiting time also increases at a rapid rate due to the limited resources.

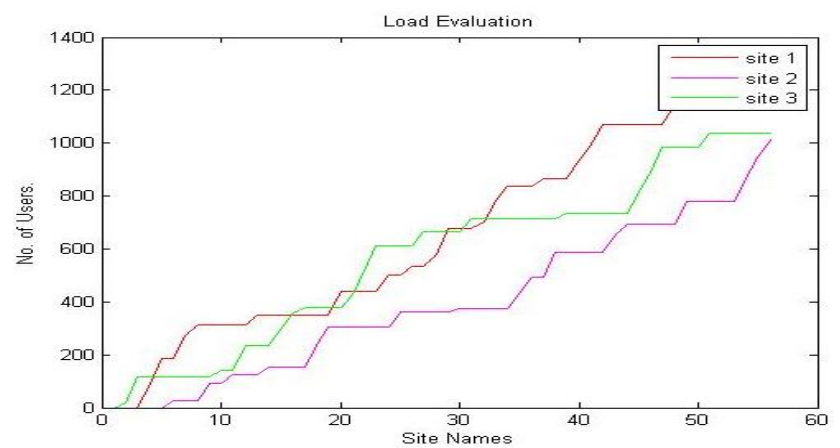

Fig. 10: Load Evaluation (Simulation time $=55$ )

"Fig. 10" shows the Load evaluation of the existing work where $y$-axis contains the number of users with respect to the server names. There are three servers refer to site 1 , site 2 and site 3 as shown in legend. Here load of site 1 is more.

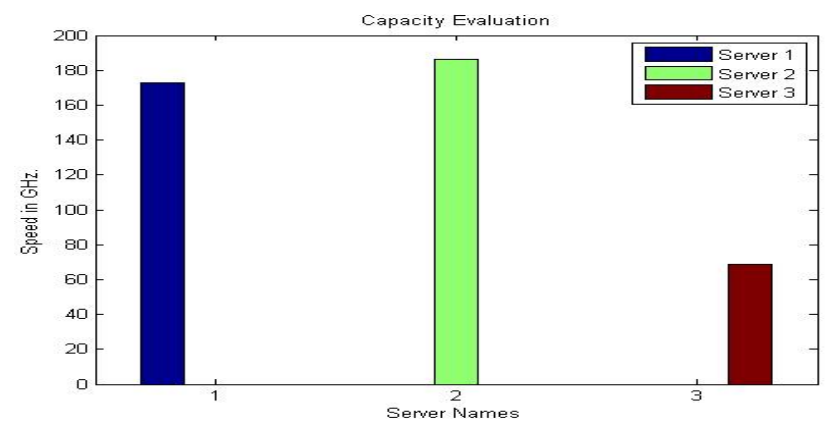

Fig. 11: Capacity Evaluation (Simulation time 55)

"Fig. 11" shows the Capacity Evaluation of the existing work where y-axis contains speed in Ghz with respect to the server names. There are three servers refer 
to server1, server 2 and server 3 as shown in legend. Here the capacity of server 1 lies in between 176 and capacity of server 2 comes nearby 190 and capacity of server 3 comes nearby 70 . So overall in this graph the server 2 has greater capacity.

2. Case 2: Experimental Results for Proposed work when simulation time is 55 :

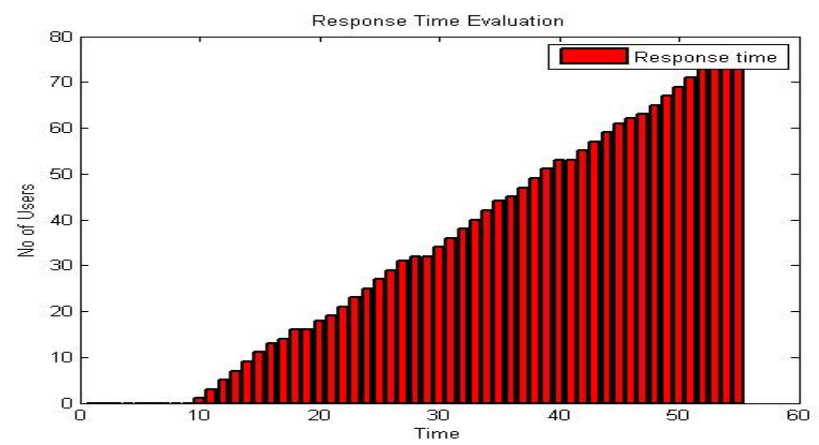

Fig. 12: Response Time Evaluation (Proposed work) (Simulation time $=55$ )

"Fig. 12" shows the response time evaluation of the proposed work where y-axis contains the number of users with respect to the time. As it is clearly showing that as the time increases response time is also increases at a rapid rate due to the limited resources.

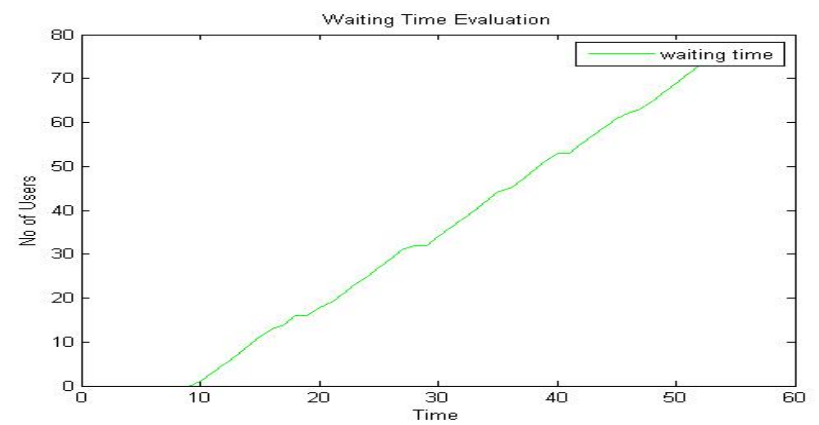

Fig. 13: Waiting Time Evaluation (Proposed work) (Simulation time $=55)$

"Fig. 13" shows the waiting time evaluation of the proposed work where y-axis contains the number of users with respect to the time. It shows that as the time increases waiting time also increases at a rapid rate due to the limited resources.

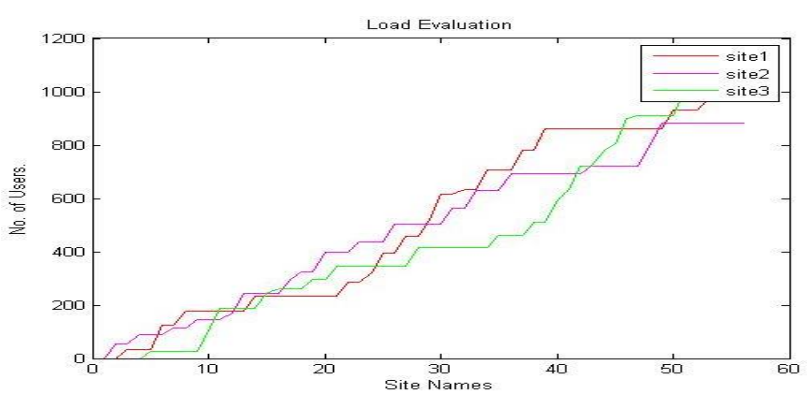

Figure 14: Load Evaluation (Proposed work) (Simulation time 55)
"Fig. 14" shows the Load evaluation of the proposed work where y-axis contains the number of users with respect to the sites names. There are three servers refer to site 1 , site 2 and site 3 as shown in legend.

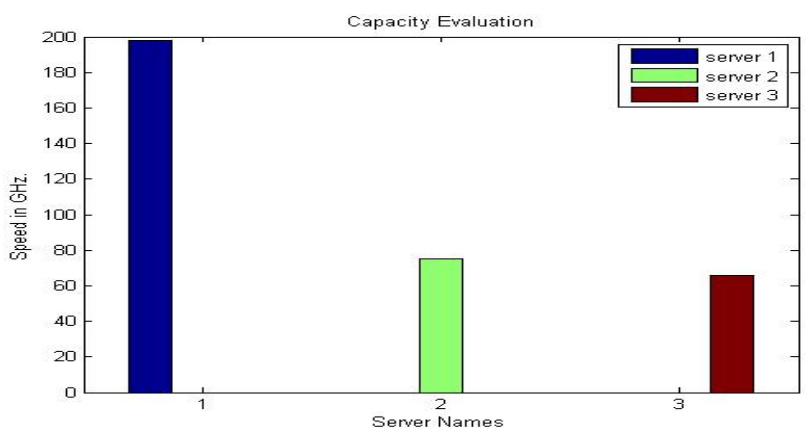

Fig. 15: Capacity Evaluation (Proposed work) (Simulation time=55)

"Fig. 15" shows the Capacity Evaluation of the proposed work where y-axis contains speed in Ghz with respect to the server names. There are three servers refer to server1, server 2 and server 3 as shown in legend. In this the server 1 has greater capacity than server 2 and server 3 .

3. Case 3: Experimental Results for Existing work when simulation time is 100 :

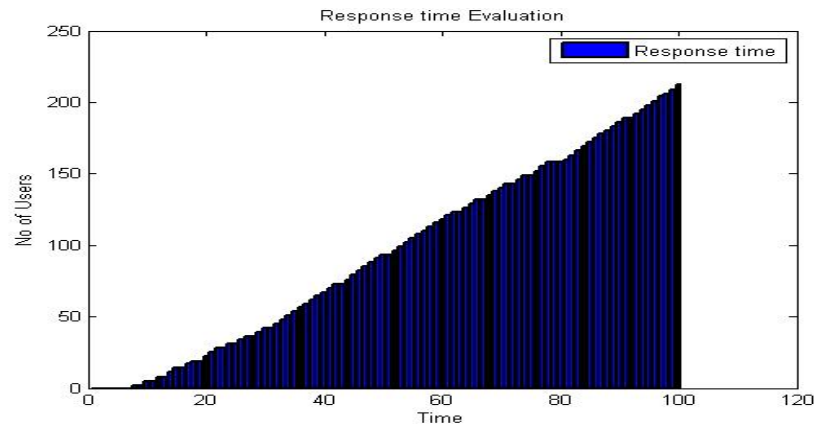

Fig. 16: Response Time Evaluation

"Fig. 16" shows the response time evaluation of the existing work where y-axis contains the number of users with respect to the time. It shows that as the time increases response time also increases at a rapid rate due to the limited resources.

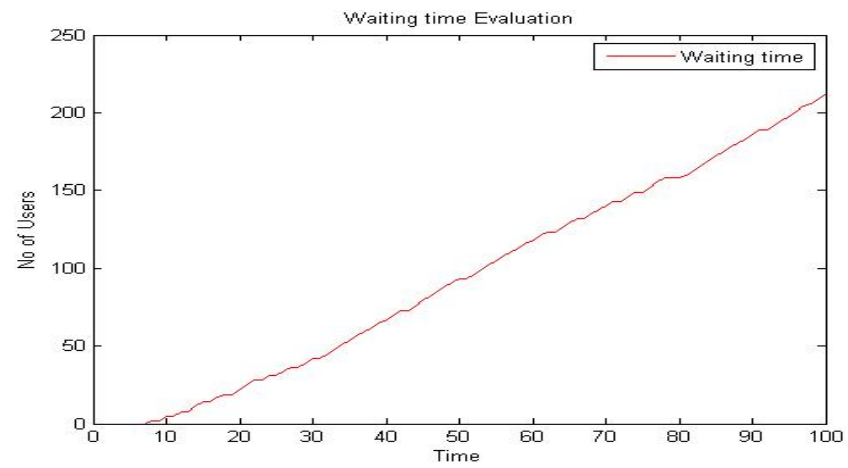

Fig. 17: Waiting Time Evaluation 
"Fig. 17" shows the waiting time evaluation of the existing work where y-axis contains the number of users with respect to the time. It shows as the simulation time increases, waiting time also increases at a rapid rate due to the limited resources.

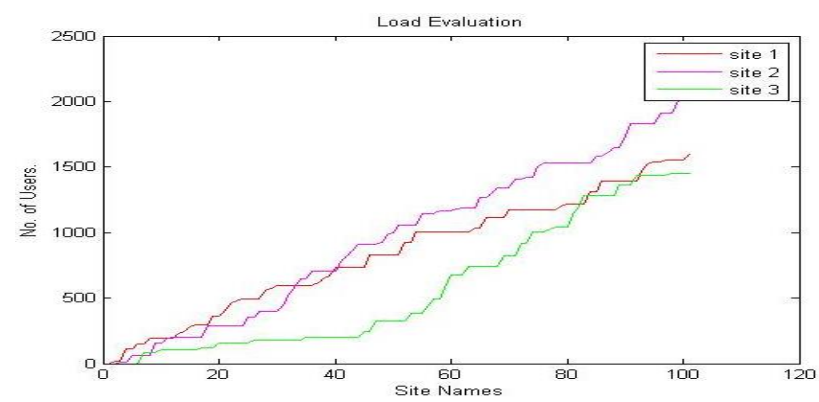

Fig. 18: Load Evaluation

"Fig. 18" shows the Load evaluation of the existing work where $y$-axis contains the number of users with respect to the sites names. There are three servers refer to site 1 , site 2 and site 3 as shown in legend. In this, site 2 has greater load as compared to other servers.

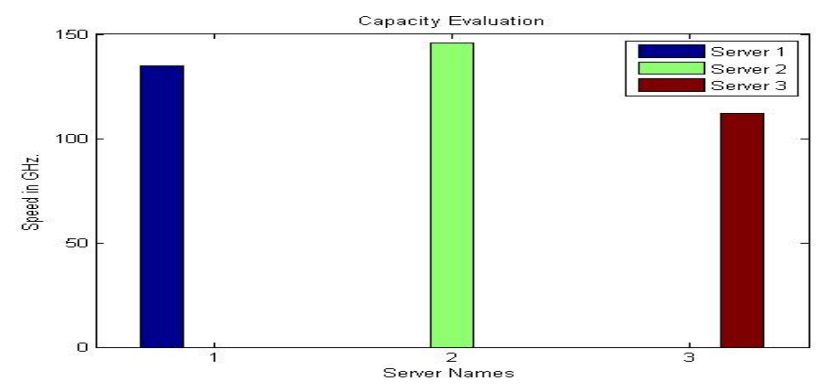

Fig. 19: Capacity Evaluation

"Fig. 19" shows the capacity evaluation of the existing work where y-axis contains the speed in $\mathrm{GHz}$ and increasing order is there with respect to the server names. There are three servers refer to server 1 , server 2 and server 3 as shown in legend. Server 2 has greater capacity as compared to other two servers.

4. Case 4: Experimental Results for Proposed work when simulation time is 100 :

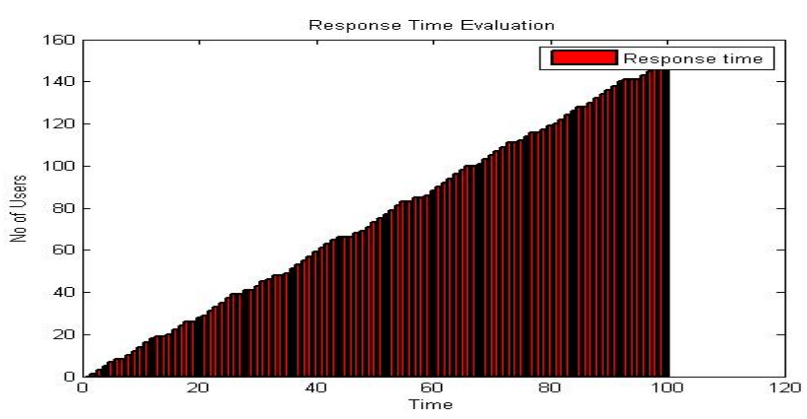

Fig. 20: Response Time Evaluation (Proposed work)
"Fig. 20" shows the response time evaluation of the proposed work where y-axis contains the number of users with respect to the time. As it is clearly showing that as the rate of simulated time increases, response time also increases at a rapid rate due to the limited resources.

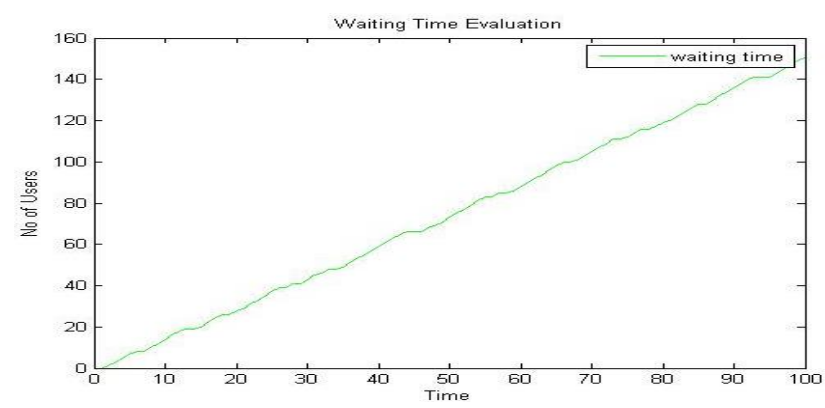

Fig. 21: Waiting Time Evaluation (Proposed work)

"Fig. 21" shows the waiting time evaluation of the proposed work where $y$-axis contains the number of users with respect to the time. It shows that as the rate of simulation time increases, waiting time also increases at a rapid rate due to the limited resources.

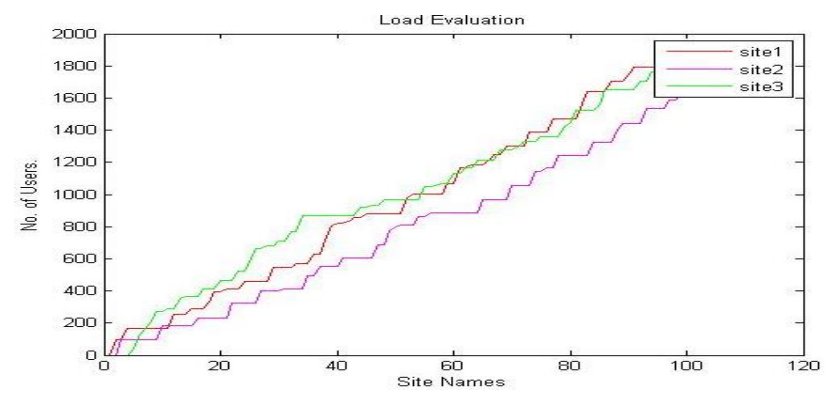

Fig. 22: Load Evaluation (Proposed work)

"Fig. 22" shows the load evaluation of the proposed work where $y$-axis contains the number of users and with respect to the site names. There are three sites such as site 1 , site 2 and site 3 . Site 1 has greater load.

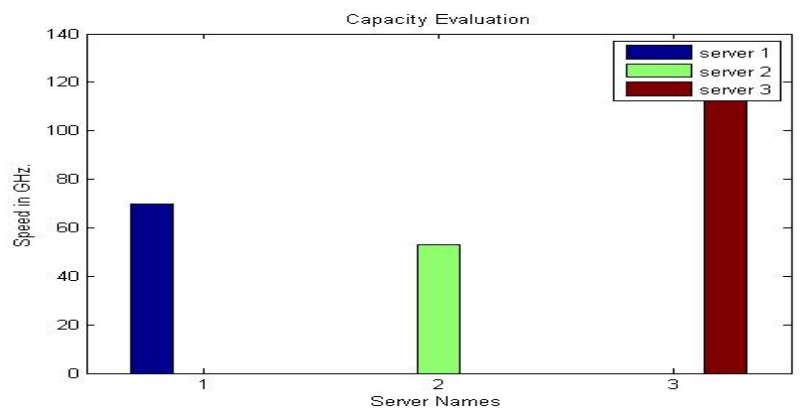

Figure 23: Capacity Evaluation (Proposed work)

"Fig. 23" show the capacity evaluation of the proposed work where $\mathrm{y}$-axis contains the speed in $\mathrm{GHz}$ with respect to the server names. It clearly shows that server 3 has greater capacity as compared to server 1 and server 2 . 
5. Case 5: Comparison of existing work and proposed work at simulation time 55

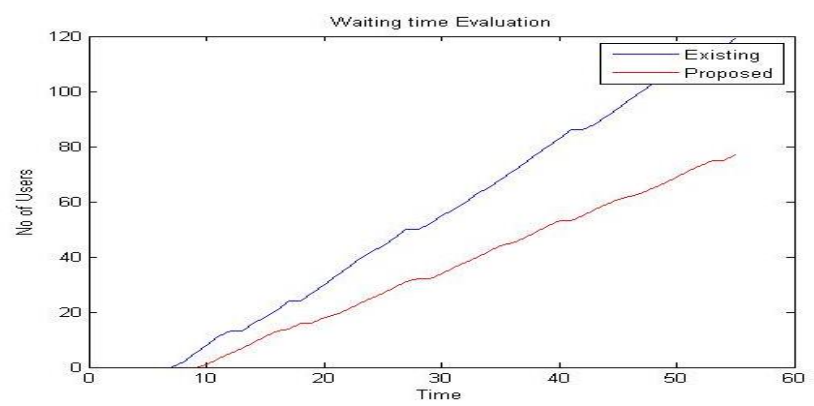

Figure 24: Waiting Time Evaluation (Existing and Proposed work) (Simulation time $=55$ )

"Fig. 24" shows the waiting time evaluation of the existing and proposed work where y-axis contains the number of users with respect to the time. As it is clearly showing that as the time increases response time is also increases at a rapid rate due to the limited resources. Here the waiting time of existing work is much than the proposed one. So, the proposed work shows better result as compared to the existing one.

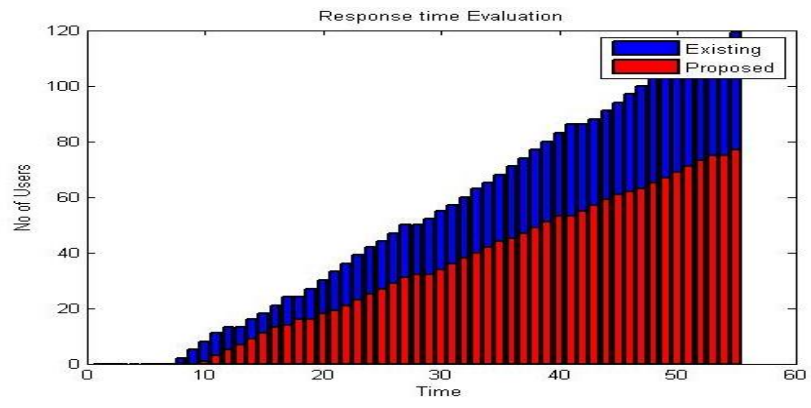

Fig. 25: Response Time Evaluation (Existing and Proposed) (Simulation time $=55$ )

"Fig. 25" shows the response time evaluation of the proposed work where y-axis contains the number of users with respect to the time. As it is clearly showing that as the time increases response time is also increases at a rapid rate due to the limited resources. Here the response time of existing work is much than the proposed one. So, the proposed work shows better result as compared to the existing one.

6. Case 6: Comparison of existing work and proposed work at simulation time 100

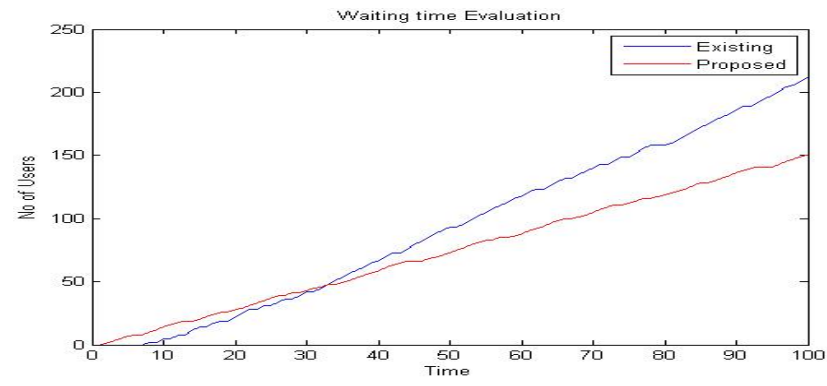

Fig. 26: Waiting Time Evaluation (Existing and Proposed work) (Simulation time $=100)$
"Fig. 26" shows the waiting time evaluation of the proposed work where y-axis contains the number of users with respect to the time. As it is clearly showing that with the time there is an increase in waiting time at a rapid rate due to the limited resources. Initially the waiting time increases but after some time it decreases. Here the waiting time of existing work is much than the proposed one. So, the proposed work shows better result as compared to the existing one.

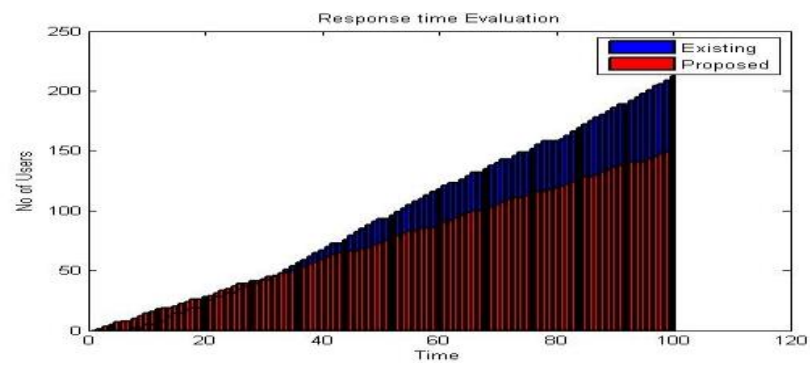

Fig. 27: Response Time Evaluation (Existing and Proposed work) $($ Simulation time $=100)$

"Fig. 27" shows the response time evaluation of the proposed work where $y$-axis contains the number of users with respect to the time. As it is clearly showing that as the time increases response time also increases at a rapid rate due to the limited resources. Here the response time of existing work is much than the proposed one. So, the proposed work shows better result as compared to the existing one.

\section{CONCLUSION AND FUTURE WORK}

This research work has proposed a novel technique to evaluate the performance power aware cloud computing using dynamic wakeup calls. The objective of this research work is to schedule workload into servers in a cost-efficient manner by avoiding occurrences when devices are under-utilized. This work has enhanced the existing work by introducing the dynamic wake up calls either to shut down the active servers or to restart the passive server. The live migration strategy is used for migration of virtual machines from one to other server according to the priorities assigned to the servers. Due to the limitation of the real time environment simulation has been done in MATLAB. The proposed algorithm results have shown that it has ability to successfully minimize the response and waiting time than the existing techniques.

\section{REFERENCES}

[1] P. Mell and T. Grance, "The NIST Definition of Cloud Computing”, NIST Report, July 2009.

[2] Y. Jadeja and K. Modi, "Cloud computing - concepts, architecture and challenges", In Computing, Electronics and Electrical Technologies (ICCEET), pp. 877-880, March 2012.

[3] Bhushan Lal Sahu and Rajesh Tiwari, "A Comprehensive Study on Cloud Computing", International Journal of Advanced Research in Computer Science and Software Engineering, Volume 2, Issue 9, September 2012. 
[4] Sarojadevi K and Jeevitha R, "Uncloud the Cloud of Cloud Computing" http://www.caesjournals.org /spluploads/IJCAES-AISC- 2011-174.pdf.

[5] Shengmei Luo, Zhaoji Lin, Xiaohua Chen, Zhuolin Yang and Jianyong Chen "Virtualization security for cloud computing service," International Conference on Cloud and Service Computing, pp.174-179, 2011.

[6] J E Smith and Ravi Nayar, "Introduction to virtual Machines", Elsevier Science, Nov 14, 2004.

[7] Q. Zhang, L. Cheng, and R. Boutaba, "Cloud computing: state-of-the-art and research challenges", Internet Services and Applications-Springer, pp.7-18, 2010.

[8] M. B. Mollah, K. R. Islam, and S. S. Islam "Next generation of computing through cloud computing technology" CCECE, page 1-6, IEEE, 2012.

[9] Shyam Patidar, Dheeraj Rane and Pritesh Jain, "A Survey Paper on Cloud Computing", Second International Conference on Advanced Computing \& Communication Technologies- IEEE, 2012.

[10] Rich Lee and B. Jeng, "Load Balancing Tactics in Cloud", International Conference on Cyber-Enabled Distributed Computing and Knowledge Discovery- IEEE, pp. 447 454, Oct. 2011.

[11] Pushtikant Malviya and Shailendra Singh, "A Study about Green Computing", International Journal of Advanced Research in Computer Science and Software Engineering, Volume 3, Issue 6, June 2013.

[12] Mrs .Sharmila Shinde, Mrs. Simantini Nalawade,Mr .Ajay Nalawade, "Green Computing: Go Green and Save Energy", International Journal of Advanced Research in Computer Science and Software Engineering, Volume 3, Issue 7, July 2013.

[13] Andrew J. Younge et al.," Efficient Resource Management for Cloud Computing Environments, IEEE, 2010.

[14] Anton Beloglazov and Rajkumar Buyya, "Energy Efficient Resource Management in Virtualized Cloud Data Centers", 10th IEEE/ACM International Conference on Cluster, Cloud and Grid Computing, 2010.

[15] Massoud Pedram, "Energy-Efficient Datacenters", To appear in IEEE Trans. On Computer Aided Design, Vol. 31, No. 10, Oct. 2012.

[16] Rajkumar Buyya, Anton Beloglazov, and Jemal Abawajy, "Energy-Efficient Management of Data Center Resources for Cloud Computing: A Vision, Architectural Elements, and Open Challenges".

[17] Andreas Berl et.al., "Energy-Efficient Cloud Computing", Published by Oxford University Press on behalf of The British Computer Society, Vol. 53 No. 7, 2010.

[18] Anubha Jain, Manoj Mishra, Sateesh Kumar Peddoju , Nitin Jain, "Energy Efficient Computing- Green Cloud Computing", IEEE, pp.978-982, 2013.

[19] Kalange Pooja R, "Applications of Green Cloud Computing in Energy Efficiency and Environmental Sustainability" IOSR Journal of Computer Engineering (IOSR-JCE), pp. 25-33.

[20] James William Smith and Ian Sommerville, "Understanding tradeoffs between Power Usage and Performance in a Virtualized Environment", IEEE Sixth International Conference on Cloud Computing, 2013.

[21] Qiang Huang, Fengqian GAO and Rui Wang, Zhengwei, "Power Consumption of Virtual Machine Live Migration in Clouds", Third International Conference on Communications and Mobile Computing, IEEE, 2011.

[22] Kejiang Ye, Xiaohong Jiang, Dawei Huang, Jianhai Chen and Bei Wang, "Live Migration of Multiple Virtual Machines with Resource Reservation in Cloud Computing
Environments", 4th International Conference on Cloud Computing- IEEE, 2011.

[23] Rasoul Beik, "Green Cloud Computing: An EnergyAware Layer in Software Architecture", IEEE, 2012.

[24] Clark, K. Fraser, S. Hand, J. G. Hansen, E. Jul, C. Limpach, I. Pratt, and A. Warfield, "Live migration of virtual machines", Proceedings of the 2nd Symposium on Networked Systems Design and Implementation (NSDI'05), 2005.

[25] Feng-Seng Chu, Kwang-Cheng Chen and Chen-Mou Cheng, "Toward Green Cloud Computing", ACM, 2011.

[26] Changyeon Jo, Erik Gustafsson, Jeongseok Son, and Bernhard Egger, "Efficient Live Migration of Virtual Machines Using Shared Storage", ACM, 2013.

[27] Kyong Hoon Kim, Anton Beloglazov, and Rajkumar Buyya, "Power-Aware Provisioning of Virtual Machines for Real-Time Cloud Services", Concurrency and Computation: Practice and Experience, 2011.

[28] R. Bianchini and R.Rajamony, "Power and energy management for server systems," IEEE Computer, voI.37, no. 11, pp.68-74, 2004.

[29] Prof. Riyaz A. Sheikh and Dr. U.A. Lanjewar, "Green Computing- Embrace a Secure Future", International Journal of computer Applications, vol-10-N4, November 2010.

[30] Ongaro, A. L. Cox and S. Rixner, "Scheduling I/O in Virtual Machine Monitors," VEE, Seattle, Washington, USA, 2008.

[31] https://help.ubuntu.com/community/Installation/SystemRe quirements.

[32] http://greencloud.gforge.uni.lu/index.html.

[33] C. Hsu and W. Feng, "A power-aware run-time system for high performance computing", Proceedings of the 2005 ACM IEEE conference on Supercomputing, 2005.

[34] D. Kliazovich, P. Bouvry, S.Ullah Khan, "GreenCloud-a packet level simulator of energy aware cloud computing data centers", Springer Science, 2010.

[35] Jyothi Sekhar, Getzi Jeba and S. Durga "A Survey on Energy Efficient Server Consolidation Through VM Live Migration", nternational Journal of Advances in Engineering \& Technology, IJAET, Nov 2012.

[36] Ms.Dheera Jadhwani, Mr.Mayur Agrawal, Mr.Hemant Mande, "Study of Efficient Utilization of Power using green Computing", International Journal of Advanced Computer Research, Volume-2 Number-4 Issue-6 December-2012.

[37] Chaima Ghribi, Makhlouf Hadji and Djamal Zeghlache, "Energy Efficient VM Scheduling for Cloud Data Centers: Exact allocation and migration algorithms", 13th IEEE/ACM International Symposium on Cluster, Cloud, and Grid Computing, 2013.

[38] Mohammad H. Al Shayeji and M.D. Samrajesh, "An Energy-aware Virtual Machine Migration Algorithm", International Conference on Advances in Computing and Communications, 2012.

[39] Negin Kord and Hassan Haghighi, "An Energy-Efficient Approach for Virtual Machine Placement in Cloud Based Data Centers", 5th Conference on Information and Knowledge Technology (IKT), IEEE- 2013.

[40] Jayant Baliga, Robert W. A. Ayre, Kerry Hinton, and Rodney S. Tucker, "Green Cloud Computing: Balancing Energy in Processing, Storage, and Transport", Proceedings of the IEEE, January 2011.

[41] Ch. Sai Krishna Manohar, "A Greener Approach to Cloud Computing using Virtual Migration", International Journal of Advanced Research in Computer Science and Software Engineering, IJARCSSE-2013. 
[42] Dragos Diaconescu, Florin Pop, Valentin Cristea, "Energy-aware Placement of VMs in a Datacenter", IEEE-2013.

\section{Authors' Profiles}

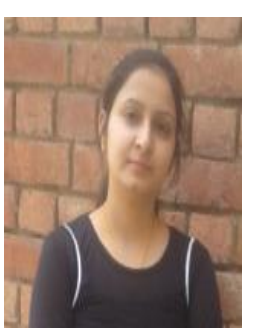

Gaganpreet Kaur received the B. Tech. degree in Computer Science from B.K.S.J Engineering College in 2012 and has done M.Tech. in Software System from Guru Nanak Dev University Campus, Amritsar respectively. Her home town is Amritsar. Her date of birth is 20.04.1990. She got first prize in project presentation and educational skit in College Level and has good knowledge of LATEX, Ms Office and C\#.net. Her area of interest is Emerging technologies.

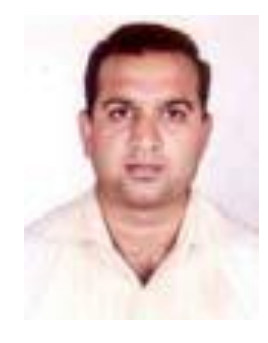

Anil Kumar has done the M. Tech. in the field of Computer Science and he is an Assistant Professor in GNDU, Amritsar. His home town is Amritsar. His areas of interest are Computer Networks, Operating System, and Computer Architecture but specialized in Computer Networks fields.

How to cite this paper: Gaganpreet Kaur Sehdev, Anil Kumar,"Performance Evaluation of Power Aware VM Consolidation using Live Migration", IJCNIS, vol.7, no.2, pp. 67-76, 2015.DOI: 10.5815/ijcnis.2015.02.08 